\title{
Performance enhancement of OFDM signals using PAPR reduction Techniques and the Comparison of their Performance
}

\author{
M.V.R.Vittal \\ Sr.AssistantProfessor, \\ G.P.R.Engg.College, \\ Kurnool,A.P,India
}

\begin{abstract}
Orthogonal frequency division multiplexing(OFDM) is a prominent technique for providing high Quality of service(QOS) to the users by mitigating the fading of signals and also for providing high data rates in multimedia services. But Peak to Average power ratio(PAPR) is a major technical challenge in OFDM which reduces the efficiency of RF Power amplifiers. In this paper, we describe the PAPR reduction techniques in OFDM signals and compare their performance.
\end{abstract}

\section{General Terms}

Algorithms, Techniques ,Comparison, Performance

\section{Keywords}

Peak to Avearge Power ratio (PAPR), Orthogonal frequency Division multiplexing(OFDM), Selected mapping(SLM), Partial Transmit sequence(PTS), DFT Spreading, Distributed FDMA(DFDMA), Localized FDMA(LFDMA)

\section{INTRODUCTION}

The growing demand for multimedia services and high data rates require the use of efficient transmission methods.Providing high quality of service(QOS) to users and mitigating the fading of signals require that Spectral efficiency must be improved and proper link reliability must be provided. Orthogonal frequency division multiplexing(OFDM) is a scheme known for its capability to reduce multipath and frequency selective fadings.

In OFDM, the high speed data stream is divided into narrowband subcarriers and these subcarriers will be orthogonal to one another.The symbol duration in OFDM is made longer by adding Cyclic prefix or Guard interval to each symbol.As long as the Cyclic prefix is longer than the Channel delay spread, OFDM offers ISI free transmission.

But Peak to Average power ratio(PAPR) has become a major technical challenge in OFDM. In this paper, we describe the OFDM system model in Section 2, introduce the concept of PAPR in section 3 and describe the PAPR reduction methods in Section 4 namely the Selected mapping, Partial Transmit sequence and DFT spreading techniques and compare their performance in Section 5.

\section{OFDM SYSTEM MODEL}

Let $\mathbf{A}=\left[\mathrm{A}_{0} \mathrm{~A}_{1} \cdots \mathrm{A}_{\mathrm{N}-1}\right]^{\mathrm{T}}$ denote an input symbol vector in the frequency domain, where $A_{k}$ represents the complex data of the $k$ th subcarrier and $N$ is the number of subcarriers. The input symbol vector is also called the input symbol sequence.The OFDM signal is generated by summing all the $N$ modulated subcarriers each of which is separated by $1 / N \mathrm{t}_{\mathrm{s}}$ in the frequency domain, where $t_{s}$ is the sampling period. Then, a continuous time baseband OFDM signal is defined as

$$
x(t)=\frac{1}{\sqrt{N}} \sum_{k=0}^{N-1} A_{k} e^{j 2 \pi \frac{k}{N t_{s}} t}, 0 \leq t<N t_{s}
$$

The Discrete time OFDM signal $\mathrm{x}(\mathrm{n})$ sampled at the Nyquist rate $\mathrm{t}=\mathrm{nt}_{\mathrm{s}}$ is defined as

$$
x(n)=\frac{1}{\sqrt{N}} \sum_{k=0}^{N-1} A_{k} e^{j 2 \pi \frac{k}{N} n}, n=0,1, \ldots \ldots . N-1
$$

Most existing PAPR reduction methods are implemented on the discrete time OFDM signals.

\section{CONCEPT OF PAPR}

Peak to Average Power ratio(PAPR) occurs due to the Summing of carriers together.The maximum peak power increases proportionally to the number of carriers in the system. After linear region, the scalar relationship is lost and the amplifier moves into saturation region. The use of amplifiers in the saturation region leads to distortions.

PAPR is expressed as

$$
\text { PAPR }=\frac{\sum_{n=0}^{N-1} \max \left\{\left|\mathrm{x}^{2}(\mathrm{n})\right|\right\}}{\mathrm{P}_{\mathrm{av}}\{\mathrm{x}(\mathrm{n})\}}
$$

Where $x(n)$ is the original signal

$\max \left[\mathrm{x}^{2}(\mathrm{n})\right]$ indicates the peak signal power

$$
\mathrm{P}_{\mathrm{av}}\{\mathrm{x}(\mathrm{n})\}=\frac{1}{N} \sum_{n=0}^{N-1} E\left\{x^{2}(n)\right\} \text { indicates the average }
$$

signal power

\subsection{Complementary CDF}

The Complementary Cumulative Distribution function (CCDF) is used to measure the probability that the PAPR of a certain data block exceeds the given threshold.

The CCDF of the PAPR of the data block is desired to compare outputs of various reduction techniques. It is defined as

$$
\begin{aligned}
\mathrm{P}(\mathrm{PAPR}>\mathrm{z}) & =1-\mathrm{P}(\mathrm{PAPR} \leq \mathrm{z}) \\
& =1-(1-\exp (\mathrm{z}))
\end{aligned}
$$




\subsection{Necessity of PAPR reduction}

Most communication systems employ Power amplifiers in the transmitter to obtain the required transmission power. For the purpose of achieving the maximum output power efficiency, the HPA is usually operated at or near the saturation region. The nonlinear characteristic of the HPA is very sensitive to the variation in signal amplitudes.The use of Power amplifiers in the Saturation region leads to distortions and introduce intermodulation products between different subcarriers. A large PAPR value increases the complexity of $\mathrm{A} / \mathrm{D}$ and $\mathrm{D} / \mathrm{A}$ converters.

All the potential benefits of OFDM transmission are reduced by high PAPR value.

\section{PAPR REDUCTION SCHEMES}

Many methods have been developed for PAPR reduction in OFDM signals[9,14]. In this section, we discuss about Selected mapping(SLM), Partial Transmit sequence(PTS) and DFT spreading techniques for reducing the PAPR in OFDM and compare their performance characteristics.

\subsection{Selected mapping(SLM) Technique}

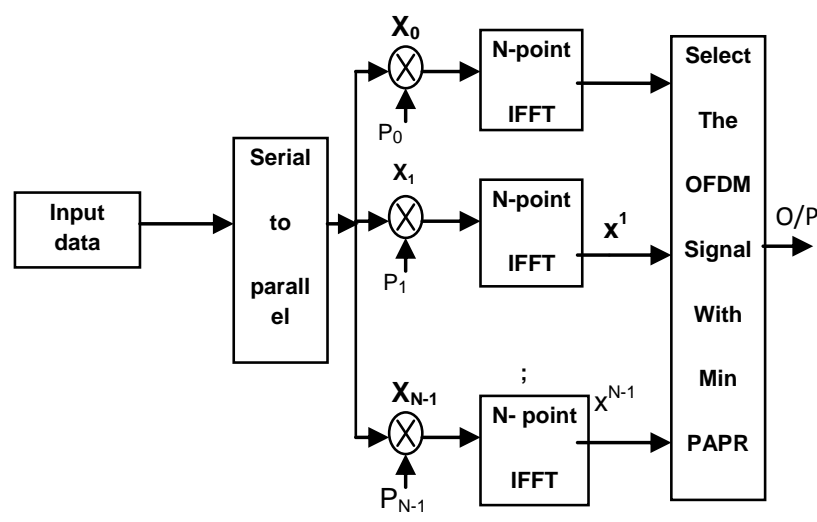

Fig 1 : Block diagram of SLM Technique

It generates the set of favourable blocks at the transmitter end which represent the original information and then chooses the most favourable block for transmission as proposed in [4]. Here the input block given by $\mathrm{X}=[\mathrm{X}(0), \mathrm{X}(1), \ldots \ldots \mathrm{X}(\mathrm{N}-1)]$ is multiplied with $\mathrm{U}$ different phase sequences $\mathrm{P}^{\mathrm{u}}=\left[\mathrm{P}_{0}{ }^{\mathrm{u}}, \mathrm{P}_{1}{ }^{\mathrm{u}}, \ldots . \mathrm{P}_{\mathrm{N}-1}{ }^{\mathrm{u}}\right]^{\mathrm{T}}$ to produce a modified data block given by

$$
\mathrm{X}^{\mathrm{u}}=\left[\mathrm{X}^{\mathrm{u}}[1], \mathrm{X}^{\mathrm{u}}[2], \ldots \ldots \mathrm{X}^{\mathrm{u}}[\mathrm{N}-1]\right]^{\mathrm{T}}
$$

The IFFT of $U$ independent sequences are taken to produce the time domain sequences $\mathrm{x}^{\mathrm{u}}=\left[\mathrm{x}^{\mathrm{u}}(0), \mathrm{x}^{\mathrm{u}}(1), \ldots . . \mathrm{x}^{\mathrm{u}}(\mathrm{N}-1)\right]^{\mathrm{T}}$ among which the one with the lowest PAPR is selected for transmission. The amount of PAPR reduction for SLM depends on the number of phase sequences $U$ and the proper design of the phase sequences[11]. In order to recover the original symbol vector in the receiver,the transmitter must send the index information about the phase sequences which is known as Side information. The implementation of SLM technique requires U IFFT operations.

\subsection{Partial Transmit sequence(PTS)}

The partial sequence(PTS) partitions an input block of $\mathrm{N}$ symbols into $\mathrm{V}$ disjoint subblocks as

$$
\mathrm{X}=\left[\mathrm{X}^{0}, \mathrm{X}^{1}, \ldots \ldots . \mathrm{X}^{\mathrm{V}-1}\right]^{\mathrm{T}}
$$

Unlike the SLM technique in which Scrambling(rotating its phase independently) is applied to each subcarrier, Scrambling is applied to each subblock in the PTS technique as specified in [5]. Then each partitioned subblock is multiplied by a complex phase vector $b v=e^{j \theta v}$ where $\mathrm{v}=1,2, \ldots . . \mathrm{V}$.IFFT is appled to each subblock to yield the following

$$
x=\operatorname{IFFT}\left\{\sum_{v=1}^{V}(b X)^{v}\right\}
$$

The above equation can be evaluated by taking the IFFT of the inner product.

Thae above equation reduces to

$$
\operatorname{IFFT}\left\{\sum_{v=1}^{V}(b X)^{v}\right\}=\sum_{v=1}^{V} b^{v} \operatorname{IFFT}\left\{X^{v}\right\}=\sum_{v=1}^{V} b^{v} x^{v}
$$

The phase vector is chosen such that the PAPR can be minimized. The corresponding time domain signal is selected such that lowest PAPR is obtained.

The selection of the phase factors is limited to a set of elements to reduce the searching complexity. The set of phase factors should be searched to find the optimum set of phase vectors. The PTS technique requires V IFFT operations for each data block. The performance of the PAPR technique depends on the number of subblocks, the number of allowed phase factors and on the subblock partitioning.

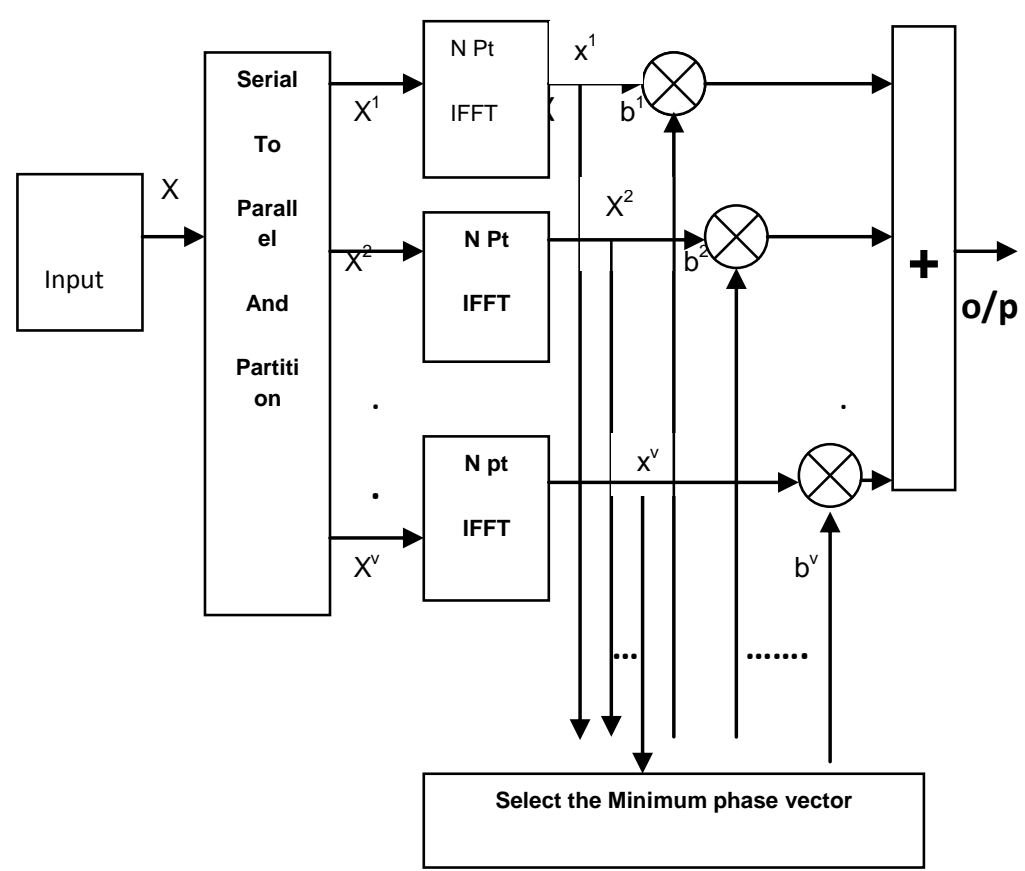

Fig 2 : Block diagram of PTS technique

The PTS technique is described with the help of the block diagram in Fig 2. Here the input data in the frequency domain is first partitioned into disjoint subblocks and then IFFT is 
applied to each subblock to find the time domain vector.Then the minimum phase vector is selected and multipled with the corresponding time domain signal and the element having least PAPR is transmitted as specified in [11]

\subsection{DFT spreading technique}

Here it is assumed that number of subcarriers allocated to each user is M. M point DFT is used for spreading and the output of DFT is assigned to the subcarriers of IFFT. The effect of PAPR depends on the way of assigning subcarriers to each terminal.
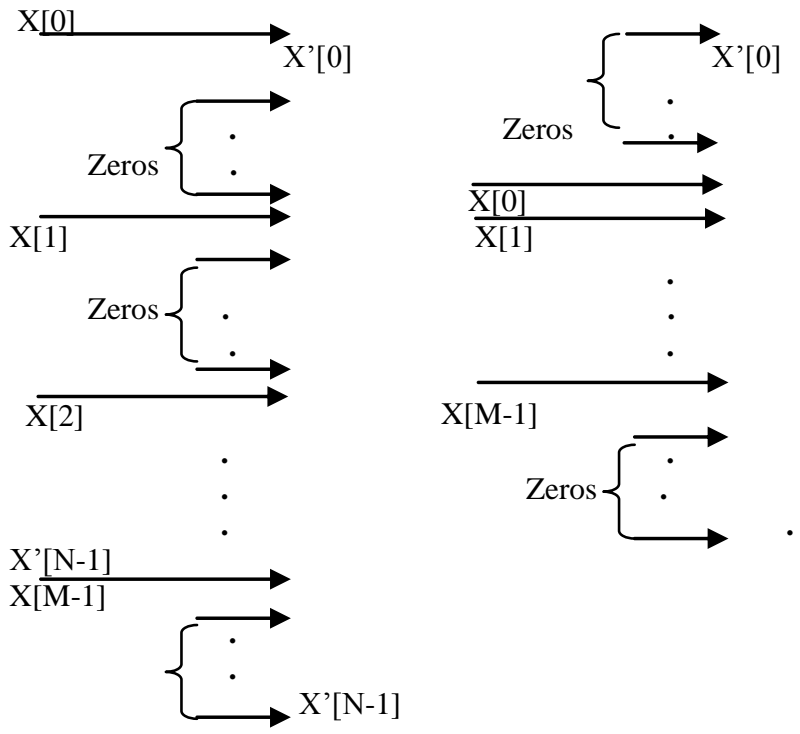

DFDMA

Fig 3: Subcarrier mapping for DFDMA and LFDMA

Fig (3) shows the subcarrier mapping for DFDMA and LFDMA. Here, DFDMA distributes M DFT outputs over the entire band of total $\mathrm{N}$ subcarriers with zeros filled in N-M unused subcarriers, whereas LFDMA allocates DFT outputs to $\mathrm{M}$ consecutive subcarriers in $\mathrm{N}$ subcarriers

In the DFT spreading technique using LFDMA, the IFFT signal $\mathrm{X}^{\prime}(\mathrm{k})$ at the transmitter can be expressed as

$$
\begin{gathered}
\mathrm{X}^{\prime}[\mathrm{k}]=\mathrm{X}[\mathrm{k}] \quad \begin{array}{l}
\mathrm{k}=0,1, \ldots . \mathrm{M}-1 \\
=0
\end{array} \quad \text { otherwise }
\end{gathered}
$$

The IFFT output sequence with $\mathrm{n}=\mathrm{S} . \mathrm{m}+\mathrm{s}$ can be expressed

$x^{\prime}[n]=x^{\prime}[S m+s]=\frac{1}{N} \sum_{k=0}^{N-1} X^{\prime}[k] e^{j 2 \pi k \frac{n}{N}}$

The above equation can be written a

$x^{\prime}[n]=\frac{1}{S} \cdot \frac{1}{M} \sum_{k=0}^{M-1} X[k] e^{j 2 \pi k \frac{S m+s}{S M}}$

For $\mathrm{s}=0$ the above equation becomes

$x^{\prime}[n]=x^{\prime}[S m]=\frac{1}{S} \cdot \frac{1}{M} \sum_{k=0}^{M-1} X[k] e^{j 2 \pi k \frac{m}{M}}=\frac{1}{S} x[m]$

(12)

For s not equal to Zero the equation 11 becomes

$$
x^{\prime}[n]=x^{\prime}[S m+s]=\frac{1}{S}(A) x[m]
$$

Here $\mathrm{A}$ is a quantity which indicates the phase factor.

From the above equations (12) and (13), it is seen that the LFDMA signal becomes $1 / \mathrm{S}$ times the scaled version of the input signal in time domain for $\mathrm{s}=0$ and $\mathrm{s}$ not equal to 0 .

\subsubsection{Pulse Shaping}

Pulse shaping using Raised Cosine filter is taken after IFFT stage for LFDMA technique. Pulse shaping is used to minimize Inter symbol Interference(ISI) and results in better transmission of

OFDM signals. The optimum value of Roll off factor $\alpha$ is chosen for obtaining better Simulation results.

\section{RESULTS AND DISCUSSION}

The PAPR reduction techniques discussed in this paper namely Selected mapping(SLM), Partial Transmit sequence(PTS) and DFT spreading technique using Localized FDMA have been simulated to compare their performance characteristics.

Table 1 : Simulation results for PTS Technique (N=128)

\begin{tabular}{|c|c|c|c|c|}
\hline $\begin{array}{c}\text { No of } \\
\text { Subblocks }\end{array}$ & 2 & 4 & 8 & 16 \\
\hline PAPR(dB) & 10 & 8.8 & 8.2 & 7.6 \\
\hline
\end{tabular}

Table 2 : Simulation results for PTS Technique $(\mathrm{N}=256)$

\begin{tabular}{|c|c|c|c|c|}
\hline $\begin{array}{c}\text { No of } \\
\text { Subblocks }\end{array}$ & 2 & 4 & 8 & 16 \\
\hline PAPR(dB) & 9.6 & 8.7 & 8.1 & 7.3 \\
\hline
\end{tabular}

Table 3 : Simulation results for SLM Technique $(\mathrm{N}=\mathbf{1 2 8}, \mathbf{2 5 6}, \mathbf{5 1 2})$

\begin{tabular}{|c|c|c|c|}
\hline $\begin{array}{c}\text { No of } \\
\text { Subblocks }\end{array}$ & 128 & 256 & 512 \\
\hline PAPR(dB) & 8.7 & 7.8 & 7.2 \\
\hline
\end{tabular}

Table 4 : Simulation results for LFDMA Technique $(\alpha=0.6)$

\begin{tabular}{|c|c|}
\hline & $\operatorname{PAPR}(\mathrm{dB})$ \\
\hline 16 QAM & 7.8 \\
\hline 64 QAM & 8.3 \\
\hline
\end{tabular}


Table 1 and Table 2 show the Simulation results for the Partial Transmit sequence(PTS) technique for two cases(Number of subcarriers $\mathrm{N}=256$ and $\mathrm{N}=128$ ). It is observed that as the number of subblocks increases, PAPR decreases.

Table 3 shows the Simulation results for Selected mapping (SLM) technique for three cases $(\mathrm{N}=128,256,512)$.It is seen that as the number of subcarriers increases, PAPR reduces.

Table 4 shows the Simulation results for DFT spreading Localized FDMA(LFDMA) technique. The simulation is carried out for two types of digital modulations namely the 16 QAM and 64 QAM.The Roll off factor ' $\alpha$ ' is chosen to be 0.6.The Simulation results show that the PAPR is low for 16 QAM compared to 64 QAM.

The Simulation results are carried out taking into account various simulation parameters like Total number of Subcarriers, Number of subblocks, Number of subcarriers allocated, Spreading factor, Roll-off factor, Type of modulation technique, Complementary CDF.

\section{CONCLUSION}

In this paper, the concept of PAPR in OFDM signals is discussed. The PAPR reduction techniques like Selected mapping(SLM), Partial Transmit sequence(PTS) and DFT spreading technique using Localized FDMA have been investigatated. The Simulation results show that as the PAPR reduces with these techniques, they can be used in OFDM transmitter effectively. The performance of RF Power amplifiers is enhanced as the PAPR decreases.

In PTS technique as the number of subblocks increases, the PAPR decreases. In Localized FDMA(LFDMA) technique, even though the PAPR increases for 64 QAM, it can be applied for systems demanding high data rates.LFDMA technique is applied in Uplink transmission for 3GPP LTE systems.

\section{REFERENCES}

[1] Ping Xiao, Yue Xiao, "An improved Gradient based PAPR reduction method for SSK-OFDM Systems", IEICE transactions on Communictions, Vol E-94,No.12,Dec 2011

[2] Hyun-Bae Jeon, Jong-Seon No, "A Low-Complexity SLM Scheme Using Additive Mapping Sequences for PAPR Reduction of OFDM Signals", IEEE Transactions on Broadcasting, vol. 57, no. 4, December 2011.
[3] Sen-Hung Wang, Jia-Cheng Sie, "A Low-Complexity PAPR Reduction Scheme for OFDMA Uplink Systems", IEEE transactions on Wireless communications, vol. 10, no. 4, April 2011.

[4] Mahmoud Ferdosizadeh Naeiny, "Selected Mapping Algorithm for PAPR Reduction of Space-Frequency Coded OFDM Systems Without Side Information", IEEE transactions on Vehicular technology, vol. 60, no. 3, March 2011.

[5] Pooria Varahram, Wisam F. Al-Azzo, “A Low Complexity Partial Transmit Sequence Scheme by Use of Dummy Signals for PAPR Reduction in OFDM Systems", IEEE Transactions on Consumer Electronics, Vol. 56, No. 4, November 2010.

[6] Yajun wang, C.Tellambura, "A PAPR reduction method based on Artificial bee colony algorithm for OFDM signals" IEEE Transactions on Wireless Communications, Vol No.9, No.10,Oct 2010.

[7] Jung-Chieh Chen, “Partial Transmit Sequences for PAPR Reduction of OFDM Signals with Stochastic Optimization Techniques", IEEE Transactions on Consumer Electronics, Vol. 56, No. 3, August 2010

[8] V.B.Malode,D.B.Patil, "PAPR reduction using modified selective mapping technique", I.J of Advanced Networking and applications, Vol.2,No.2,Jan 2010.

[9] Hyun-Jeon,Jong seon, "Bit based SLM schemes for PAPR reduction in QAM modulated OFDM signals", IEEE transactions on Broadcasting,Vol.55,No.3,Sep.2009.

[10] Jing Gao, Wang,Zhibin, "A novel concurrent algorithm for STBC MIMO-OFDM systems",I.J. of Information and System sciences, Vol.4,No.3,2008.

[11] Tao Jiang,Yiyan Wu, “An overview of PAPR reduction techniques for OFDM signals",IEEE transactions on Broadcasting,Vol.54,No.2,June 2008.

[12] Seok-Joong, Dong-Joon, "A modified SLM scheme with low complexity for PAPR reduction of OFDM systems", IEEE transactions on Broadcasting,Vol.53,No.4,Dec 2007.

[13] Dae-woon,Seok-Joong, "A new PTS scheme with low complexity for PAPR reduction",IEEE transactions on Broadcasting, Vol.52,No.1,Mar.2006

[14] A.D.Jayalath,C.Tellambura, "SLM and PTS peak power reduction of OFDM signals without side information", IEEE transactions on Wireless communications,Vol.4,No.5,Sep.2005 